\title{
Probabilistic Anatomic Mapping of Cerebral Blood Flow Distribution of the Middle Cerebral
}

\section{Artery}

Seong-Jang Kim ${ }^{1,2}$, In-Ju Kim ${ }^{1,2}$, Yong-Ki Kim ${ }^{1,2}$, Tae-Hong Lee ${ }^{2,3}$, Jung Sub Lee ${ }^{2,4}$, Sungmin Jun ${ }^{1}$, Hyun-Yeol Nam ${ }^{1}$, Jae Sung $\mathrm{Lee}^{5}$, Yu Kyeong Kim${ }^{5}$, and Dong Soo Lee, $\mathrm{PhD}^{5}$

${ }^{1}$ Department of Nuclear Medicine, Pusan National University Hospital, Busan, Republic of Korea; ${ }^{2}$ Medical Research Institute, Pusan National University Hospital, Busan, Republic of Korea ${ }^{3}$ Department of Radiology, Pusan National University Hospital, Busan, Republic of Korea; ${ }^{4}$ Departement of Orthopaedic Surgery, Pusan National University Hospital, Busan, Republic of Korea; and ${ }^{5}$ Department of Nuclear Medicine, Seoul National University, Seoul, Republic of Korea

Probabilistic atlases are more representative of the population than single brain atlases. They allow anatomic and functional labeling of the results of group studies in stereotactic space and, hence, the automated anatomic labeling of individual brain imaging data. Methods: In the current study, probabilistic maps of the blood flow distribution of the middle cerebral artery (MCA) were developed using the basal and MCA brain SPECT images. Twenty-nine patients (mean age $\pm S D, 54.6 \pm 6.1$ y) who previously received placement of a stent for MCA stenosis (right MCA stenosis, 15 patients; left MCA stenosis, 14 patients) were included in the current study. Of the 29 MCA SPECT images, 18 were analyzed for the final result because 11 MCA SPECT images revealed an uneven uptake distribution of ${ }^{99 m T c-e t h y l c y s-~}$ teinate dimer in the brain. MCA brain SPECT images were coregistered to basal brain SPECT images, and spatial normalization parameters used for basal brain SPECT images were reapplied to MCA brain SPECT for anatomic standardization. Pixel counts of the MCA brain SPECT images were then normalized, and the probabilistic map of cerebral perfusion distribution (perfusion probabilistic map) for each hemisphere was determined by averaging the spatial- and count-normalized MCA brain SPECT images. Population-based probabilistic maps representing the extent of MCA territory (extent probabilistic map) were also composed by averaging the binary images obtained by thresholding the spatially normalized MCA brain SPECT images. Results: The blood supply from the MCA to the basal ganglia area was largest (probability, $0.6 \sim 0.8$ ), followed by the insular cortex (probability, 0.3 0.5), and various cerebral cortical areas (probability, $0.2 \sim 0.4$ ). The MCA reached to deep structures of the brain, including the internal capsule, caudate nucleus, putamen, globus pallidus, insular cortex, and thalamus with a high-extent probability. Conclusion: A population-based probabilistic map of MCA flow distribution was generated by using MCA brain SPECT images. This map could be a potential tool for the analysis of major cerebral artery distribution, especially the MCA. Furthermore, the probabilistic MCA atlas could be

Received Jul. 29, 2007; revision accepted Oct. 12, 2007.

For correspondence or reprints contact: Yong-Ki Kim, MD, PhD, Department of Nuclear Medicine and Medical Research Institute, Pusan National University Hospital, Busan, 602-739, Republic of Korea.

E-mail: yongki@pusan.ac.kr

COPYRIGHT @ 2008 by the Society of Nuclear Medicine, Inc. used to define the object delineation of the MCA territory, to quantify ischemic disease affecting the MCA, to predict prognosis, and to risk stratification of cerebrovascular diseases, especially affecting the MCA.

Key Words: probabilistic map; middle cerebral artery; SPECT; statistical parametric mapping

J Nucl Med 2008; 49:39-43

DOI: 10.2967/jnumed.107.045724

$\mathbf{T}$ he advent of modern mathematic and computational approaches to averaging imaging data across subjects has led to a generation of population-based probabilistic atlases (1). Population-based brain atlases offer a powerful framework to synthesize the results of disparate imaging studies. Also, these atlases allow the user to obtain relative information that takes into account the variance in structure and function of the human population.

PET and SPECT can effectively and noninvasively measure cerebral metabolism and cerebral blood flow. Using the spatial normalization and probabilistic maps of the brain, PET and SPECT brain images have been used for investigation of the perfusion and metabolic abnormalities in various diseases $(2,3)$.

Recently, a population-based probabilistic brain map of the blood flow distribution of the internal carotid artery (ICA) was established using amobarbital SPECT (4). This newly developed vascular system map could be a potential and important instrument for the assessment of various intracranial cerebrovascular diseases.

The middle cerebral artery (MCA) is one of the 3 major paired arteries that supply blood to the brain. It is one of the most complex intracranial cerebral arteries and is commonly affected by cerebrovascular accidents. The MCA supplies most of the outer brain surface, nearly all of the basal ganglia, and the posterior and anterior internal capsule (5). 
For clinical implications, accurate and precise knowledge of the perfusion territories of the cerebral arteries is essential, and the delineation of individual cerebral artery flow territories is useful in evaluation of the mechanism of stroke and aids the planning of investigation and subsequent treatment. However, available maps of cerebral arterial territories are largely perfusion maps based on injection studies in cadavers $(6-8)$.

Therefore, the absence of a clear definition of the MCA territory has encouraged an alternate strategy for the development of probabilistic maps of this territory based on perfusion images. In this study, we developed the populationbased probabilistic anatomic map of the cerebral blood flow distribution of the MCA, which might have a crucial role in the assessment of cerebrovascular diseases.

\section{MATERIALS AND METHODS}

\section{Patients}

Twenty-nine patients (mean age $\pm \mathrm{SD}, 54.6 \pm 6.1 \mathrm{y}$ ) who received stent placement previously for MCA stenosis (right MCA stenosis, 15 patients; left MCA stenosis, 14 patients) were included in this study. Because cerebral angiography is an extremely invasive procedure, a population of normal healthy individuals could not be recruited. We excluded patients who had major complications, such as a major cerebral infarction or hemorrhage of the nonaffected cerebral hemisphere, chronic complete obstruction of the MCA, stenosis distal to the M1 bifurcation of the nonaffected artery, previous head trauma, psychiatric illness, alcoholism and drug abuse, epilepsy, and kidney, liver, or lung failure. Before placement of the MCA stent, the patients with MCA stenosis showed recurrent transient ischemic attacks or cerebral infarction despite continuation of antiplatelet agents and statin. Each patient gave informed consent and underwent a neurologic examination and the MiniMental State Examination, with normal results.

\section{Basal and MCA 99mTc-Ethylcysteinate Dimer (99mTc-ECD) Brain SPECT}

Basal and MCA brain SPECT images were acquired using a commercially available dual-head $\gamma$-camera (Vertex; Philips) with a low-energy, high-resolution collimator. Before basal SPECT, all subjects were in the supine position, with their eyes closed, in a quiet room with lights dimmed. The basal SPECT image was obtained 15 min after injection of $750 \mathrm{MBq}$ of ${ }^{99 \mathrm{~m}} \mathrm{Tc}-\mathrm{ECD}$. The energy window was set at $140 \mathrm{keV}$ with a symmetric $10 \%$ window width. One hundred twenty-eight frames were acquired for $15 \mathrm{~s}$ per frame. The images $(128 \times 128$ matrices $)$ were reconstructed with the filtered backprojection method using a Butterworth filter. All images were corrected for attenuation using Chang's method (9). MCA brain SPECT was performed during the follow-up angiography for the evaluation of in-stent restenosis. The time interval between basal and MCA brain SPECT was $4.8 \pm 1.75 \mathrm{~d}(2 \sim 9 \mathrm{~d})$. The patients were premedicated with a daily dose of $100 \mathrm{mg}$ aspirin and $75 \mathrm{mg}$ clopidogrel (Plavix; Sanofi-Synthelabo) for at least $2 \mathrm{~d}$ before the follow-up cerebral angiography. In addition, 2,850 IU/0.3 $\mathrm{mL}$ of low-molecular-weight nadroparin calcium (Fraxiparine; Sanofi-Synthelabo) were injected subcutaneously 2 or 3 times a day during the same period. The patient was fully awake during the procedure. The electrocardiogram, arterial oxygen saturation, and blood pressure were appropriately monitored. After confirmation of the absence of in-stent restenosis, the MCA SPECT procedure was performed on the contralateral nonaffected MCA. For MCA SPECT, a 6- to 7-French guiding catheter (Envoy; Cordis Endovascular Corp.) was positioned in the proximal portion of the M1 portion of the MCA. Through the guiding catheter, a 0.014-in microcatheter (Transend; Boston Scientific Corp.) was positioned in the proximal M1 portion of the MCA. After visual inspection of the angiographic absence of leptomeningeal anastomosis with the anterior cerebral artery and the posterior communicating cerebral artery and the absence of flow to anterior or posterior communicating cerebral arteries, $750 \mathrm{MBq}$ of ${ }^{99 \mathrm{~m}} \mathrm{Tc}-\mathrm{ECD}$ were injected into catheter and flushed with $5 \mathrm{~mL}$ of normal saline. After this procedure, hemostasis of the femoral artery was achieved using an occlusion device (Angioseal; St. Jude Medical). Follow-up brain CT was immediately obtained for the evaluation of acute hemorrhagic complications. Two hours after completion of the angiography, the subjects were moved to the nuclear medicine section, and then MCA brain SPECT images were acquired using the same protocol as that for basal brain SPECT. The brain MR images, including a diffusion-weighted image and gradient-recalled echo of the subjects, as well as complete neurologic examinations were obtained within $3 \mathrm{~d}$ after the procedure.

\section{Anatomic Standardization}

Of 29 MCA SPECT images, 18 were analyzed for the final result because 11 MCA SPECT images revealed an uneven uptake distribution of ${ }^{99 \mathrm{~m}} \mathrm{Tc}-\mathrm{ECD}$ in the brain, which was determined visually under the consensus of 3 experts. For the anatomic standardization of the MCA SPECT data, we followed the procedures used for generating the ICA probabilistic maps in our previous study (4). First, the MCA image of each individual was manually registered with the corresponding basal brain SPECT image using the FIRE program (Functional Image Registration; Seoul National University, Seoul, Korea) (10). Only the rigid-body transformation with 6 degrees of freedom was allowed in this registration. Boundaries of the brain cortex and of the subcortical structures with high activity (i.e., basal ganglia and anterior/posterior temporal area) in both brain SPECT images were used as anatomic landmarks for the manual registration. The basal brain SPECT image was then spatially normalized onto the standard brain perfusion SPECT template, and the normalization parameter of the basal SPECT image was then reapplied to the MCA SPECT image. Statistical parametric mapping 5 (SPM5; Institute of Neurology, University College of London, U.K.) was used for the spatial normalization.

\section{Perfusion Probabilistic MCA Map}

The perfusion and extent probabilistic maps were generated using mirrored images of the injected cerebral hemispheres to increase the reliability of the probabilistic maps by increasing the sample size. We assumed that distributions of the MCA blood supply in both hemispheres were similar. All spatially normalized MCA SPECT images were split into 1-side images (left or right), and only the images of the injected cerebral hemisphere were mirrored. Images of the other side were not used. To generate the perfusion probabilistic maps, the count normalizations of the mirrored images were performed and the normalized images were averaged as they were done (4). The pixel values of the images were normalized with respect to the maximum pixel value in each image (count normalization) to remove the effects of different injection doses and individual differences in the global uptake of 
${ }^{99 m}$ Tc-ECD in the brain. After the count normalization, perfusion probabilistic maps were composed by averaging the spatial- and count-normalized MCA brain SPECT images.

\section{Extent Probabilistic MCA Map}

An extent probabilistic MCA map was also generated following the procedures used (4). However, the threshold values for the segmentations of images were adjusted to the characteristics of the MCA images. To obtain the binary image of MCA territory of each individual, first, the initial global MCA mask were generated by thresholding the perfusion probabilistic map; the threshold was $40 \%$ of the maximum pixel value in the perfusion probabilistic map. The threshold of $40 \%$ was selected because the contour line with this threshold matched best with the cortical boundary of the T1-weighted (T1) MRI template of SPM. Applying the MCA mask onto the individual SPECT images, mean pixel values in the masked regions and $50 \%$ of the mean values were obtained. These values were applied to the individual images that were not masked to obtain the individual binary image of the MCA territory. The threshold of $50 \%$ was also selected using the same criteria. In addition, no region with ${ }^{99 \mathrm{~m}} \mathrm{Tc}-\mathrm{ECD}$ uptake higher than that of background was lost in the binary image with this threshold. Finally, the binary images were averaged to obtain the extent probabilistic map of the MCA territory.

\section{RESULTS}

Figure 1A shows the perfusion probabilistic map of MCA generated by averaging the mirrored MCA SPECT images after the count normalization. The probabilistic map was superimposed on the standard T1 MRI template provided in the SPM program. Only the data $>0.1$ of the normalized count are visualized. The perfusion probabilistic map represents the cerebral blood flow rate rather than the extent of MCA vascular territory. The blood supply from the MCA to the basal ganglia area was largest (probability, $0.6 \sim 0.8$ ), followed by the insular cortex of the temporal lobe (probability, $0.3 \sim 0.5$ ), and various cerebral cortical areas (probability, 0.2 0.4). The cortical blood from the MCA was found to reach a region from the frontal lobe to as far as the parietooccipital and temporooccipital junction, including the middle occipital gyrus. Of these various cortical areas, superior and middle temporal gyri and middle and inferior frontal gyri were found to have a high likelihood of being supplied by the MCA, whereas the posterior part of the middle temporal gyrus, the inferior temporal gyrus, showed a low likelihood. Also, the superior frontal gyrus, the midbrain including the pons, and the occipital gyrus did not receive the blood supply from the MCA. The SD map of the normalized MCA SPECT images is shown in Figure 1B, which represents the regional variability in the MCA perfusion.

Figure 2 shows the extent probabilistic map of the MCA territory. The MCA supplies blood to the broad regions of brain. It supplies blood flow to the frontal lobe anteriorly, including the middle and inferior frontal gyri except the superior frontal gyrus. Also, the MCA reached to deep structures of the brain, including the internal capsule, caudate nucleus, putamen, globus pallidus, insular cortex, and thalamus with a high-extent probability (probability, $0.6 \sim 1.0)$. However, the blood supply to the posterior part
A

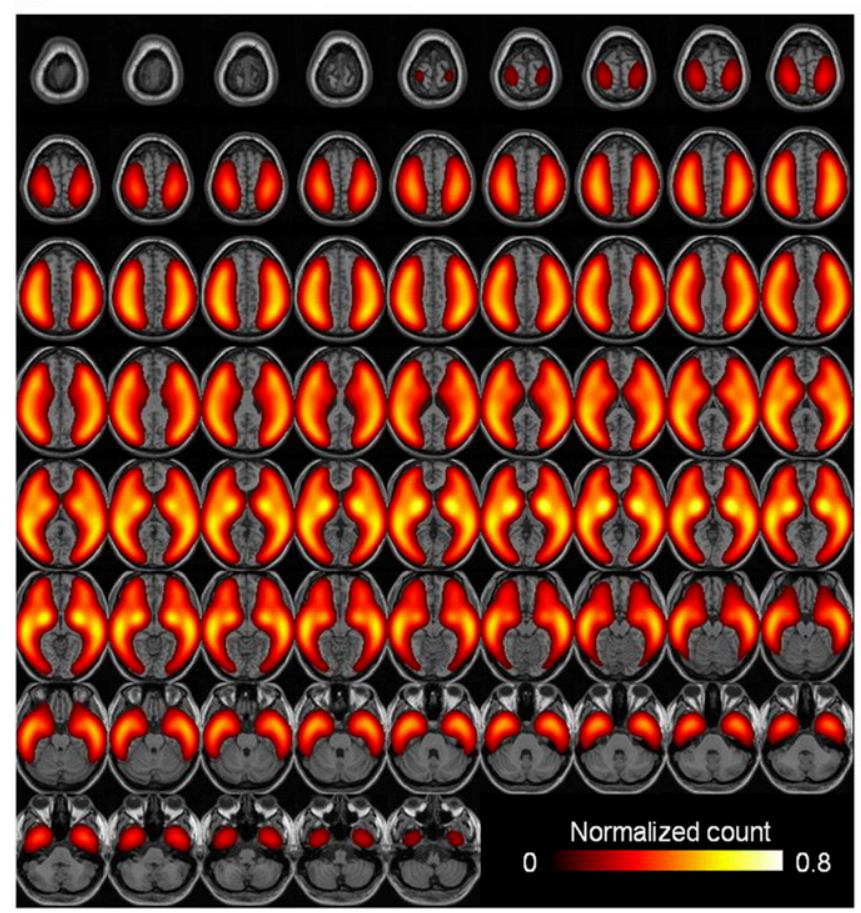

B

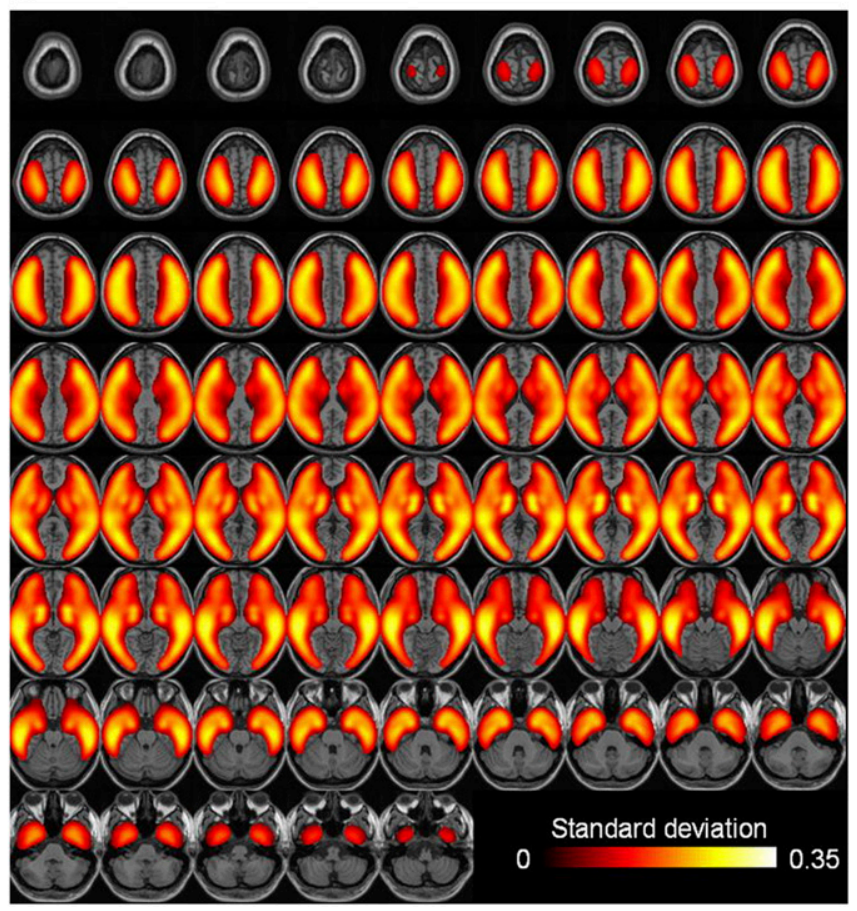

FIGURE 1. (A) Perfusion probabilistic map of MCA superimposed on standard T1 MRI template. Perfusion probabilistic map represents cerebral blood flow rate of MCA vascular territory. Probability of blood supply from MCA to basal ganglia area was the largest. (B) SD map of MCA perfusion. 


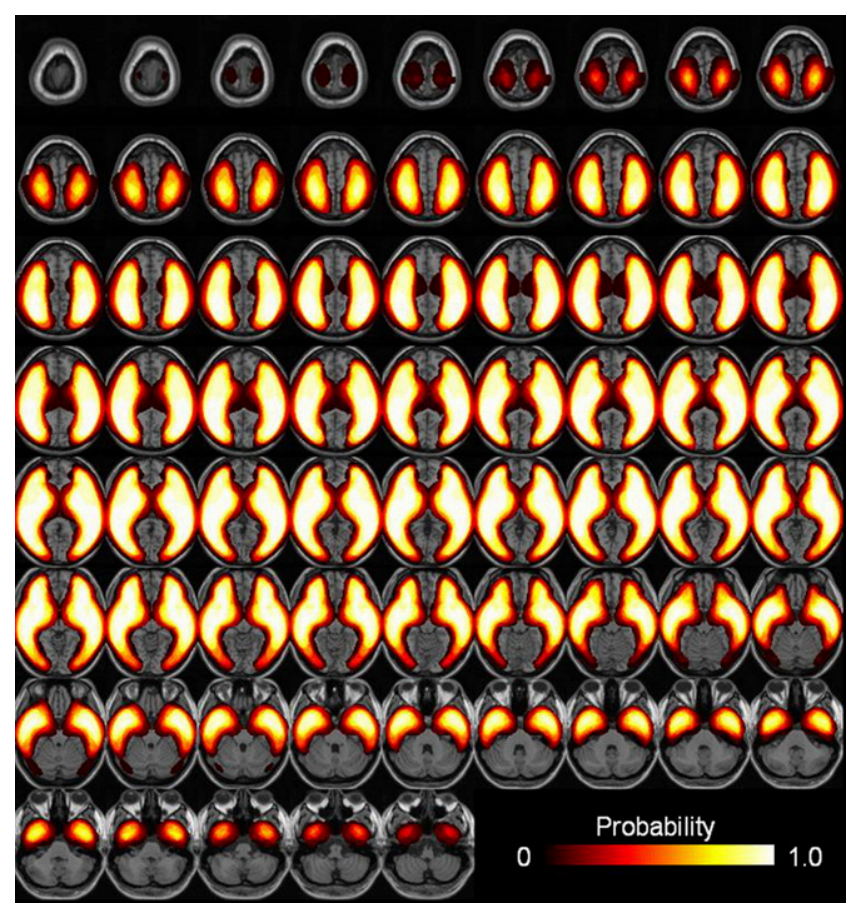

FIGURE 2. Extent probabilistic map for vascular territory of MCA. MCA reached to deep structures of brain, including internal capsule, caudate nucleus, putamen, globus pallidus, insular cortex, and thalamus with a high-extent probability.

of the brain, including the posterior temporal region and the occipitoparietal, and temporooccipital area, was low (probability, <0.4). In the extent probabilistic map, the MCA did not reach to the areas of the superior frontal gyrus, the midbrain including pons, and the occipital lobe. Also, the MCA did not supply the blood flow to the interhemispheric fissure of the brain.

\section{DISCUSSION}

Population-based probabilistic maps use novel analytic devices to fuse individually various data and are powerful new tools with broad clinical and research applications, including pediatric disorders and degenerative tissue loss rates in normal aging and dementing diseases (11-14).

The current study generated 2 different probabilistic atlases of the MCA. A probabilistic atlas of the MCA was made from individual binary contours of its territory. Each voxel on the MCA SPECT map belongs to the MCA territory in probabilistic terms. Therefore, this atlas represents regional cerebral blood flow distributions from the MCAnamely, an extent probabilistic map. Little variation of the extent from the MCA was noted in the deep structure of the brain, especially in the basal ganglia. However, the blood supply to the posterior part of the brain, including the posterior temporal region and the occipitoparietal, and temporooccipital area, was low, and the probability of the extent of the MCA varied. Also, the extent probabilistic map showed that the superior frontal gyrus and the occipital lobe did not receive their blood supply from the MCA. These findings are different from those of earlier investigations in that the MCA territory was shown to reach the occipital poles $(8,15)$.

A previous study showed that, in most cases, the anterior cerebral artery (ACA)-MCA boundary on the superior lateral surface of the hemisphere was found in the superior frontal sulcus, followed by the superior frontal and middle frontal gyri, and, lastly, the inferior frontal sulcus (7). Also, the MCA posterior cerebral artery (PCA) boundary on the lateral and inferior surface of the temporal lobe was recorded primarily in the inferior temporal gyrus (7).

Another probability atlas generated from this study was the map of the vascular contribution from the MCA and represented the fractional supply from the MCA according to the tissue demand as well as the territorial variability. This perfusion probability map of the MCA represents the cerebral blood flow rate rather than the extent of the MCA vascular territory. Among various cerebral structures, the subcortical structure of the basal ganglia showed the highest likelihood of being supplied by the MCA, followed by the insular cortex, and various cerebral cortices. This highest probability of the basal ganglia could be explained by the fact that the perforating branches of the MCA usually originate from the M1 portion (16). These perforating branches supply the superior part of the caudate nucleus, the lateral segment of the globus pallidus, the putamen, the dorsal half of the internal capsule, and the lateral half of the anterior commissure (17).

The cortical blood from the MCA was found to reach a region from the frontal lobe to as far as the parietooccipital and temporooccipital junction. Of these various cortical areas, the superior and middle temporal gyri and the middle and inferior frontal gyri were found to have a high likelihood of being supplied by the MCA. However, the posterior part of the middle temporal and the inferior temporal gyri showed a low likelihood. Also, the superior frontal gyrus, the midbrain including pons, and the occipital gyrus did not receive their blood supply from the MCA.

Recently, flow territory mapping of major cerebral feeding arteries has been conducted (18). Despite the merit of this in vivo experiment, selective spin labeling of MRI could result in the possibility of contamination of arteries from other vasculature (18). A digital map of MCA infarcts using T2-weighted (T2) MRI was also established (19). This digital map demonstrated that the regions with the highest probability of MCA infarction were the striatocapsular area, the centrum semiovale, and the insular cortex, which is similar to the findings of the current study. However, this digital map did not represent the authentic perfusion map; rather, it shows the MCA-associated map.

The currently developed MCA probabilistic map might have some potential clinical implications. First, it could be used for quantification of MCA infarction using a volumeof-interest method and follow-up after revascularization treatment using surgery or intraarterial stent placement. 
Furthermore, this map would have a potential role for prognostication of patients with MCA stenosis or infarction. Second, as the MCA probabilistic map is 3-dimensional, it could be used to overlay results of group comparisons, giving probability-based information. Third, the stereotaxically normalized MCA probabilistic map contains the probabilistic information for any given voxel of the MCA flow territory. Fourth, this map could be used for individual analysis of the MCA territory.

However, limitations of the current study should be considered. We found that the MCA reached to deep structures of the brain, including the internal capsule, caudate nucleus, putamen, globus pallidus, insular cortex, and thalamus with a high-extent probability. However, it is well known that the thalamus derives its blood supply from several arteries, including the polar and paramedian arteries, the inferolateral (thalamogeniculate) arteries, and the posterior (medial and lateral) choroidal arteries. These arteries are branches of the posterior cerebral artery. One possible explanation could be due to poor spatial resolution of the brain perfusion SPECT images. The present SPECT image may not separately delineate lenticular nuclei and thalamus.

\section{CONCLUSION}

In the current study, using MCA brain SPECT images, a population-based probabilistic map of the MCA flow distribution was generated. This map could be another potential tool for analysis of the major cerebral artery distribution, especially the MCA. Furthermore, the probabilistic MCA atlas could be used to define the object delineation of the MCA territory, to quantify ischemic disease affecting the MCA, and to predict prognosis and to stratify the risk of cerebrovascular diseases, especially affecting the MCA.

\section{ACKNOWLEDGMENTS}

This study was supported by a grant from the Medical Research Institute of Pusan National University (grant 2006-42) and, in part, by a grant from the Korea Ministry of
Science and Technology Radiation Applied Neuroscience (grant M2 050407000405A070700410).

\section{REFERENCES}

1. Mazziotta JC, Toga AW, Evans A, Fox O, Lancaster J. A probabilistic atlas of the human brain: theory and rationale for its development. Neuroimage. 1995;2: 89-101.

2. Kang E, Lee DS, Lee JS, et al. Developmental hemispheric asymmetry of interregional metabolic correlation of the auditory cortex in deaf subjects. Neuroimage. 2003;19:777-783.

3. Lee DS, Lee JS, Kang KW, et al. Disparity of perfusion and glucose metabolism of epileptogenic zones in temporal lobe epilepsy demonstrated by SPM/SPAM analysis on $\left[{ }^{15} \mathrm{O}\right]$ water PET, $\left[{ }^{18} \mathrm{~F}\right] \mathrm{FDG}-\mathrm{PET}$, and $\left[{ }^{99 \mathrm{~m}} \mathrm{Tc}\right]-\mathrm{HMPAO}$ SPECT. Epilepsia. 2001;42:1515-1522.

4. Lee JS, Lee DS, Kim YK, et al. Probabilistic map of blood flow distribution in the brain from the internal carotid artery. Neuroimage. 2004;23:1422-1431.

5. Moore KL, Dalley AR. Clinically Oriented Anatomy. 4th ed. Philadelphia, PA: Lippincott Williams \& Wilkins; 1999.

6. van der Zwan A, Hillen B. Review of the variability of the territories of the major cerebral arteries. Stroke. 1991;22:1078-1084.

7. van der Zwan A, Hillen B, Tulleken CA, Dujovny M, Dragovic L. Variability of the territories of the major cerebral arteries. J Neurosurg. 1992;77:927-940.

8. Tatu L, Moulin T, Bogousslavsky J, Duvernoy H. Arterial territories of the human brain: cerebral hemispheres. Neurology. 1998;50:1699-1708.

9. Chang LT. A method of attenuation correction in radionuclide computed tomography. IEEE Trans Nucl Sci. 1978;25:638-643.

10. Lee JS, Park KS, Lee DS, Lee CW, Chung JK, Lee MC. Development and applications of a software for Functional Image Registration (FIRE). Comput Methods Programs Biomed. 2005;78:157-164.

11. Kikinis R, Shenton ME, Iosifescu DV, et al. A digital brain atlas for surgical planning, model-driven segmentation, and teaching. IEEE Trans Vis Comput Graph. 1996;2:232-241.

12. Roland PE, Zilles K. Brain atlases: a new research tool. Trends Neurosci. 1994; 17:458-467.

13. Thompson PM, Hayashi KM, de Zubicaray G, et al. Dynamics of gray matter loss in Alzheimer's disease. J Neurosci. 2003;23:994-1005.

14. Sowell ER, Thompson PM, Peterson BS, Welcome SE, Henkenius AL, Toga AW. Mapping cortical change across the human life span. Nat Neurosci. 2003; 6:309-315.

15. Caviness VS, Makris N, Montinaro E, et al. Anatomy of stroke. Part I. An MRIbased topographic and volumetric system of analysis. Stroke. 2002;33:25492556.

16. Marinkovic SV, Kovacevic MS, Marinkovic JM. Perforating branches of the middle cerebral artery: microsurgical anatomy of their extracerebral segments. J Neurosurg. 1985;63:266-271.

17. Umansky F, Gomes FB, Dujovny M, et al. The perforating branches of the middle cerebral artery: a microanatomical study. J Neurosurg. 1985;63:261-265.

18. van Laar PJ, Hendrikse J, Golay X, Lu H, van Osch MJP, van der Grond J. In vivo flow territory mapping of major brain feeding arteries. Neuroimage. 2006; 29:136-144.

19. Phan TG, Donnan GA, Wright PM, Reutens DC. A digital map of middle cerebral artery infarcts associated with middle cerebral artery trunk and branch occlusion. Stroke. 2005;36:986-991. 